\title{
Disclination Loops as Fundamental Excitations in Three-Dimensional Active Matter
}

1. Topological structure and dynamics of three-dimensional active nematics Authors: G. Duclos, R. Adkins, D. Banerjee, M. S. E. Peterson, M. Varghese, I. Kolvin, A. Baskaran, R. A. Pelcovits, T. R. Powers, A. Baskaran, F. Toschi, M. F. Hagan, S. J. Streichan, V. Vitelli, D. A. Beller, and Z. Dogic

Science 367, 1120 (2020)

\section{Three-Dimensional Active Defect Loops}

Authors: J. Binysh, Ž. Kos, S. Čopar, M. Ravnik, and G. P. Alexander Phys. Rev. Lett. 124, 088001 (2020)

\section{Recommended with a Commentary by Jonathan V. Selinger, Kent State University}

The concept of active matter describes systems of interacting objects that consume energy and transform it into movement [1,2]. This concept was originally developed to describe biological systems, such as flocks of birds or schools of fish, in which the individual animals interact with each other and develop collective patterns of motion. Over the past 10-20 years, it has been applied to a wide range of systems, including swarms of bacteria, growth of epithelial tissue, and nonbiological systems such as self-propelled colloidal particles.

In many active systems, the interactions between particles lead to orientational order, analogous to the orientational order of a magnet or a liquid crystal. Often this order is nematic, which means that particles are oriented forward and backward along some axis, as in the most common liquid-crystal phase. However, unlike conventional nematic liquid crystals, active nematics do not relax to some equilibrium state that minimizes the free energy. Rather, they are continually in motion, swirling around, so that the material is flowing and the pattern of orientational order is changing.

In any experiment on active matter, or in any simulation, many different dynamic processes are occurring at the same time. It is difficult to decide what to observe, or how to interpret the data. For that reason, it is important for researchers to develop some reduced description of what is happening within an active system, using a limited number of degrees of freedom. In that respect, the study of active matter is similar to the study of other many-body systems, in which physicists seek to identify quasiparticles as the fundamental excitations to be studied.

So far, most studies have concentrated on two-dimensional (2D) active nematics. In these 2D systems, it is useful to study a class of topological point defects, known as disclinations, as the relevant quasiparticles. Disclinations have the structure shown in Fig. 1. Here, the gray double- 
headed arrows represent the local orientation of nematic order, called the director field $\mathbf{n}(x, y)$. This director is well-defined everywhere except at the two singular points, shown in black. The point on the right is a disclination of topological charge $+1 / 2$, because the director rotates halfway around a circle in a positive sense as one traverses the blue loop. Likewise, the point on the left is a disclination of topological charge $-1 / 2$, because the director rotates halfway around a circle in a negative sense around the blue loop. Note that the $+1 / 2$ disclination is shaped like a comet, with a characteristic vector orientation represented by the green arrow, while the $-1 / 2$ has three-fold symmetry.

While disclinations occur in both conventional and active nematic liquid crystals, their dynamic behavior is quite different in those systems. In conventional liquid crystals, disclinations of opposite charge attract each other and eventually annihilate, allowing the system to coarsen in time. By contrast, in active nematics, disclinations are continually in motion, with disclination pairs nucleating and annihilating. In particular, $+1 / 2$ disclinations induce a characteristic fluid flow pattern, which causes the disclinations to move along the direction indicated by the green arrow (either forward or backward along this arrow, depending on the type of material). Many studies have investigated this behavior through theory, simulations, and experiments. For example, one recent study has investigated how the Kosterlitz-Thouless disclination unbinding transition is modified by the presence of activity [3].

In the last two years, researchers have begun to study active nematics in 3D. At this point, the question is how to generalize the concept of disclinations as quasiparticles from $2 \mathrm{D}$ to $3 \mathrm{D}$. Naively, we might expect to extend each type of disclination out of the plane, to form a disclination line in 3D. However, this generalization is not trivial, because 3D disclination lines have very different topological properties than $2 \mathrm{D}$ disclination points. When the nematic order is $2 \mathrm{D}$, there are distinct types of disclinations with any half-integer or integer charge. However, when the nematic order is $3 \mathrm{D}$, it is possible to continuously transform $\mathrm{a}+1 / 2$ into $\mathrm{a}-1 / 2$ via twist of the director, and it is possible to continuously transform any integer disclination into a defectfree state via escape into the third dimension. Hence, from a topological perspective, there is only one type of 3D disclination line. How can we understand the behavior of 3D active nematics in terms of this one type of disclination line?

The first article discussed here, by Duclos et al., provides a combined experimental and theoretical study of that question [4]. On the experimental side, it investigates a 3D active nematic system based on microtubules driven by kinesin molecular motors, which the authors had previously studied in 2D. It uses a multiview light sheet microscope to image the system as a function of 3D position and time. Through a real tour de force of data analysis, the authors extract the director field $\mathbf{n}(x, y, z, t)$, and then identify the regions of high gradients $|\nabla \mathbf{n}|^{2}$, which are associated with disclinations. They find a network of 3D disclination lines, which spans the entire system, together with isolated disclination loops. These isolated loops nucleate and grow out of uniform regions, or contract and self-annihilate to leave uniform regions. Also, isolated 
loops merge with the larger network, or break off from the network when it selfintersects. The experimental dynamics of the material can be regarded as the dynamics of the moving disclination loops.

On the theoretical side, Duclos et al. provide a geometric method to characterize disclination loops. Geometry is more specific than topology; geometry can distinguish between objects that are topologically equivalent. Duclos et al. point out that the local structure of a disclination line can be characterized by two mutually perpendicular unit vectors: the direction $\boldsymbol{\Omega}$ about which the director
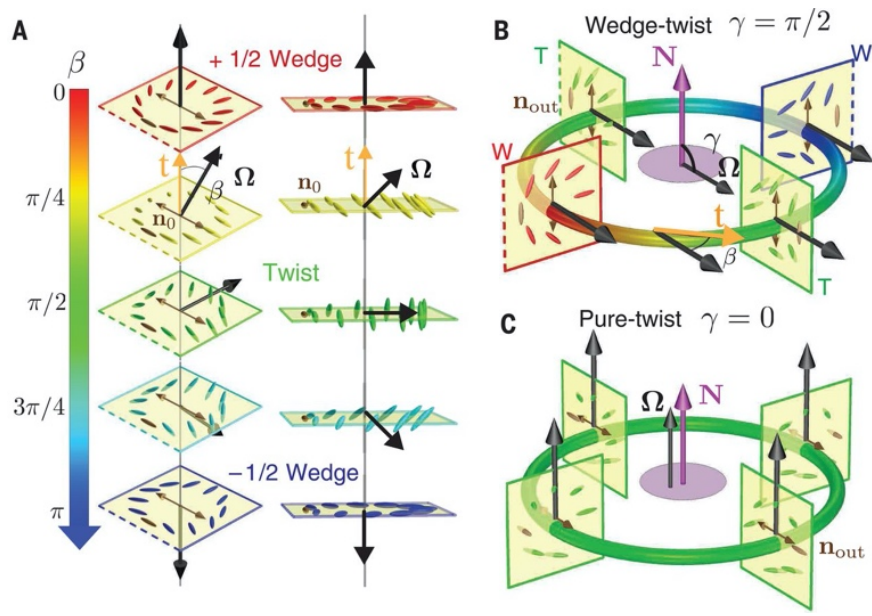

Figure 2: Possible structures of a straight disclination line $(A)$ or of a circular disclination loop $(B, C)$ in a $3 D$ nematic. From Ref. 4. field rotates, and the orientation $\mathbf{n}_{\text {out }}$ of the director just outside the loop. Based on the how these two vectors are oriented with respect to the local tangent to the loop, one can distinguish whether the local director has a $+1 / 2$ wedge (planar) structure, a twisted 3D structure, a $-1 / 2$ wedge structure, or something intermediate between these cases, as shown in Fig. 2. Moreover, based on how these vectors change around the entire length of the loop, one can characterize the topological properties of the whole loop. Certain types of loops have a higher-order topological charge, like a hedgehog in the director field, while other types of loops have no hedgehog charge. Only the loops without hedgehog charge are free to form and annihilate. Duclos et al. apply this geometric analysis to the loops observed in experiment and simulation, and show that they are consistent with this condition.

The second article discussed here, by Binysh et al., investigates the dynamics associated with disclination loops in 3D active nematics [5]. This article begins by describing the geometry of a local segment of a disclination loop using the vector $\boldsymbol{\Omega}$ and one other vector. It puts this geometry into the Stokes equation for fluid flow driven by active force, and calculates the selfpropelled velocity of this local segment. It then goes on to address the dynamic properties of the entire loop, and shows that activity might drive the loop to extend, or contract, or buckle into a nonplanar 3D shape. The type of behavior depends on the global geometry of the loop, as characterized by the global arrangement of $\boldsymbol{\Omega}$ and the other vector. These predicted behaviors are consistent with analysis of simulations for an active nematic inside a spherical droplet.

Overall, these articles demonstrate that disclination loops can be regarded as the fundamental excitations of the 3D active nematic liquid crystals. They are not exactly quasiparticles, but might be called quasi-polymers or quasi-strings. In some ways, they are analogous to a melted lattice of flux lines in a high-temperature superconductor [6]. We expect that future work may express other properties of 3D liquid crystals, either conventional or active, in terms of properties of disclination lines. For example, the elastic free energy of a 2D liquid crystal with point disclinations can be regarded as a Coulomb-like interaction between disclinations of the same or opposite topological charges. The elastic free energy of a 3D liquid crystal with one or more disclination lines might be analyzed in a similar way, as an interaction among line segments with 
certain disclination geometries. This perspective should provide further opportunities to simplify the analysis of the complex physics within active matter.

\section{References}

[1] M. C. Marchetti, J. F. Joanny, S. Ramaswamy, T. B. Liverpool, J. Prost, M. Rao, and R. A. Simha, Rev. Mod. Phys. 85, 1143 (2013).

[2] S. Ramaswamy, J. Stat. Mech. Theory Exp. 2017, 054002 (2017).

[3] S. Shankar, S. Ramaswamy, M. C. Marchetti, and M. J. Bowick, Phys. Rev. Lett. 121, 108002 (2018).

[4] G. Duclos, R. Adkins, D. Banerjee, M. S. E. Peterson, M. Varghese, I. Kolvin, A. Baskaran, R. A. Pelcovits, T. R. Powers, A. Baskaran, F. Toschi, M. F. Hagan, S. J. Streichan, V. Vitelli, D. A. Beller, and Z. Dogic, Science 367, 1120 (2020).

[5] J. Binysh, Ž. Kos, S. Čopar, M. Ravnik, and G. P. Alexander, Phys. Rev. Lett. 124, 088001 (2020).

[6] D. R. Nelson, Phys. Rev. Lett. 60, 1973 (1988). 